
\title{
$\begin{array}{ll}\text { Research Square } & \text { Preprints are preliminary reports that have not undergone peer review. } \\ \text { They should not be considered conclusive, used to inform clinical practice, } \\ \text { or referenced by the media as validated information. }\end{array}$
}

\section{IncRNA-Associated ceRNA Networks in Spleen of Nocth1-correlated T-ALL Leukemia Mice}

\author{
Min Shi \\ Second Hospital of Hebei Medical University \\ Shaohua Wang \\ Second Hospital of Hebei Medical University \\ Xiaofei Li \\ Second Hospital of Hebei Medical University \\ Yongjun Li ( sm8344@hotmail.com ) \\ Second Hospital of Hebei Medical University
}

\section{Research article}

Keywords: T-ALL, Nocth1, deep RNA-sequencing analysis, IncRNA, ceRNA

Posted Date: January 28th, 2020

DOI: https://doi.org/10.21203/rs.2.21227/v2

License: (c) (i) This work is licensed under a Creative Commons Attribution 4.0 International License. Read Full License 


\section{Abstract}

Background Acute T-lymphocytic leukemia (T-ALL) is a highly aggressive malignant tumor in leukemia. Nocth1 is considered as an major oncogene in the development of T-ALL. Increasing evidences have revealed that the occurrence and progression of T-ALL referred to abnormal gene expression, pathway activation and the regulation between these genes. However, the potential IncRNA-associated competing endogenous RNA (ceRNA) network involved in spleen of Nocth1-correlated T-ALL leukemia mice remains unclear.

Methods Overexpression of Notch intracellular domain (ICN) of Notch1 by retroviral infection was used to set up mouse T-ALL model. Deep RNA-sequencing analysis was performed the expression of IncRNAs and mRNA in spleen of T-ALL mice and C57BL/6 mice.

Results The deep RNA-sequencing analysis shown that 1833 IncRNAs and 4626 mRNAs were deregulated according to the P-value $(p<0.05)$ and fold change $(>2$-fold $)$ in spleen of T-ALL leukemia mice compared with that of C57BL/ 6 mice. Gene Ontology(GO) and KEGG pathway analysis were performed to reveal the potential roles of differentiated expressed IncRNAs. Co-expression Network was performed to reveal the regulation relationship between the differentiated expressed IncRNAs and mRNAs. CeRNA prediction constructed the IncRNA-miRNA-mRNA model to find the core ceRNA based on regression model analysis and seed sequence matching methods.

Conclusion This study provided a systematic overview of the altered IncRNAs and mRNA expression, pathway and ceRNA regulation network in the pathogenesis of Nocth1-correlated T-ALL.

\section{Background}

T-lymphocytic leukemia (T-ALL) is caused by transformation of immature T-cell progenitors, leading to abnormal proliferation in bone marrow and peripheral blood[1]. T-ALL accounts for $20-25 \%$ of the total incidence of acute lymphoblastic leukemia in adults, for $10 \%-15 \%$ of childhood acute lymphoblastic leukemia[2]. The abnormal proliferation T cell can infiltration and damage to various of tissues and affect organ function. Despite the combined chemotherapy and allogeneic hematopoietic stem cell transplantation have applied to clinical treatment of T-ALL, its event-free survival rate is only $30 \%-50 \%[3]$. Explore the mechanism and pathogenesis of T-ALL is of great significance in improving survival rate.

The formation of T-ALL is a multi-step process which includes the activation of oncogenes and inactivation of tumor suppressor genes[4]. Notch1 is a subtype of Notch receptor in Notch signaling pathway which participated in multiple pathological and physiological processes, including cancer[5]. Approximately 55\% of T-ALL patients are related with acquired functional mutations in Notch1[6]. Block Notch signaling pathway causes cell cycle arrest and apoptosis in T-ALL cell lines[7]. Notch1 is a class I transmembrane protein which transduces information from extracellular signals into nucleus directly[8]. The Notch intracellular domain (ICN) of the Notch1 is its active component, which can activate expression of target genes in nucleus. Overexpressed of ICN1 by retroviral infection in hematopoietic progenitor cells or thymocytes promote T ALL tumorigenesis, which was made to set up mouse TALL model[9].

IncRNAs are a special class of non-coding RNAs (ncRNAs), participated with varies of physiological cellular processes as well as cancer pathological process[10]. Increasing evidences have revealed that IncRNAs play a critical role in many types of cancers, including hepatocellular carcinoma, renal cell carcinoma and leukemia[11]. Investigating IncRNAs specifcally transcriptional profiles in T-ALL is of great significance to understand the gobal 
altered expression of IncRNAs. IncRNAs have diverse mechanism to regulate gene expression. Some studies show that IncRNAs act as competing endogenous RNAs (ceRNA) through a IncRNA-miRNA-mRNA model to regulate gene expression[12]. Based on the IncRNAs and mRNAs specifcally transcriptional profiles, construction the ceRNA network can enrich the raw data in studying the potential mechanism in the formation of T-ALL.

In the present study, we performed the deep RNA-sequence to analyze expression profile in spleen of T-ALL leukemia mice with the aim of explore the IncRNAs catalogue. We showed the differentially expressed IncRNAs and mRNAs. Gene Ontology(GO) and KEGG pathway analysis were performed to reveal the potential roles of these mRNAs. Coexpression Network was performed to reveal the regulation relationship between the differentially expressed IncRNAs and mRNAs. Meanwhile, we performed ceRNA prediction to construct the IncRNA-miRNA-mRNA model. Thus, this study provided a systematic overview of the altered RNAs expression, pathway and ceRNA network in the pathogenesis of Nocth1-correlated T-ALL.

\section{Methods}

\section{Animals and tissues}

We used T-ALL leukemia mice overexpressing the Notch I intracellular domain (NICD) as the research model. T-ALL leukemia cells presented by the Institute of Hematology, Peking Union Medical College, Chinese Academy of Medical Sciences. We cotransfected with retroviral plasmid MSCV-ICN1-IRES-GFP (ICN1-GFP), the reverse transcription packaging protein CMV-VSVG and and Kat into 293T. C57BL/6 mice were divided into two groups randomly, T-ALL mice and C57BL/ 6 mice, and each group has three mice. T-ALL mice group were treated as follows, collected the viral supernatant to infect C57BL/ 6 mouse bone marrow cells (Lin-Scal ${ }^{+}$), and then transplanted into C57BL/ 6 mice $\left(10^{6}\right.$ per mouse)after semi-lethal dose irradiation through tail vein injection. C57BL/ 6 mice group were transplanted culture medium in the same way. Mice were numbered, divided into three cages randomly and reared routinely. C57BL/6 mice, 6 8 weeks female, were purchased from Charles River Experimental Animal Technology Co., Ltd. (Beijing, China). 2 weeks after, the mice were sacrificed by cervical dislocation and removed the spleen by laparotomy(Fig.1A). Mice quarantined in a $12 \mathrm{~h}$ light and $12 \mathrm{~h}$ dark photoperiod pathogen free environment, received water and food in clean class animal room of Hebei Medical University. All animals were housed and cared in accordance with the Declaration of Helsinki and the guidelines and regulations of the Institutional Animal Care and Use Committee of the Second Hospital of Hebei Medical University.

\section{RNA-Seq}

RNeasy mini kit (Qiagen, Germany) was used to isolate the total RNA. TruSeq ${ }^{\text {TM }}$ RNA Sample Preparation Kit (Illumina, USA) was used to synthesize paired-end library according to TruSeq ${ }^{\text {TM }}$ RNA Sample Preparation Guide. Qubitß 2.0 Fluorometer (Life Technologies, USA) was used to quantified the purified libraries and Agilent 2100 bioanalyzer (Agilent Technologies, USA) was used to confirm the insert size and calculate the mole concentration. Illumina NovaSeq 6000 (Illumina, USA) was used to sequenced the cluster (10 pM) generated by cBot. The library construction, sequencing and bioinformatics analysis were performed at Shanghai Sinomics Corporation.

\section{RNA extraction.}

The spleen was homogenized. Total RNA was isolated by E.Z.N.A.® Total RNA Kit I following the manufacturer's Guide. Total RNA were quantifed by Nanodrop 2000 (Nanodrop Technologies, Wilmington, USA) and Agarose gel electrophoresis. RNAs with a $260: 280$ ratio of $\geq 1.5$ and 28 S: 18 S ratio is approximately 2:1. 


\section{qRT-PCR}

Differently expressed IncRNAs were selected for validation by qRT-PCR. Total RNA was reverse-transcribed using SureScript ${ }^{\mathrm{TM}}$ First-Strand cDNA Synthesis Kit (Genecopoeia, USA) according to the manufacturer's guide. qPCR reactions was performed using SYBR-Green (Invitrogen) according to the manufacturer's guide. BioRad iQ5 RealTime thermocycler was used to perform qPCR reactions. The Cycling conditions was denaturing at $95^{\circ} \mathrm{C}$, followed by 39 cycles of $95^{\circ} \mathrm{C}(10 \mathrm{~s})$ and $55^{\circ} \mathrm{C}$ annealing $(30 \mathrm{~s})$. Specific primers of each IncRNA were listed in Table 2.

\section{Statistical analysis}

The differentially expression levels of IncRNAs and mRNAs in T-ALL mice or C57BL/ 6 mice spleen was analyzed by Bioconductor package (limma version 3.26.1) and R (version 3.2.2) software. Spearman correlation test was used to analyze co-expression relationships between the IncRNAs. CeRNA prediction was analyzed by Pearson's correlation coefficients. qRT-PCR data was shown as the means \pm S.E.M. Differences between two groups were analyzed by Student's $t$ test. $\mathrm{P}<0.05$ was considered as significantly.

\section{Results}

\section{Deep RNA-sequence IncRNAs and mRNA expression profiles in spleen of T-ALL mice.}

Before injection the mice were healthy and two weeks later, deep RNA-sequence was performed the expression of IncRNAs and mRNA in spleen of T-ALL mice (3 samples) and C57BL/6 mice (3 samples). Compare with control mice, 475 IncRNAs were upregulated and 1398 IncRNAs were downregulated according to the P-value $(p<0.05)$ and fold change (>2-fold). Among of them, 89 IncRNAs and 386 IncRNAs were upregulated or downregulated significantly differentially expressed ( $>10$-fold). The differentially expressed IncRNAs were converted into the volcano plot (Fig.1BB) and scatter plot(Fig.1C) to show the differentially expressed IncRNAs visibly. Compared with the spleen of C57BL/ 6 mice, noncancerous tissues mRNAs expression profiles, 2041 IncRNAs or 3585 IncRNAs were upregulated or downregulated differentially expressed ( $p<0.05,>2$-fold). Among them, 252 IncRNAs or 999 IncRNAs were upregulated or downregulated significantly differentially expressed (>10-fold)(Fig.1DE).

\section{2. mRNA GO and KEGG pathway analysis}

In order to clarify the biological processes, cellular components and molecular functions of differentially expressed mRNAs, we performed GO terms enrichment and KEGG pathway analysis. The GO terms enrichment for differentially expressed mRNAs were related to melanocyte differentiation, myosin complex and translation repressor activity in biological processes(Fig.2A), cellular component(Fig.2B) or molecular function(Fig.2C), respectively. KEGG pathway analysis showed that showed that 303 pathways were significantly enriched among the transcripts(Fig.2D). Acute myeloid leukemia, protein export and glycosaminoglycan biosynthesis - keratan sulfate were the 3 significantly enriched pathways.

\section{Coding/non-coding co-expression analysis}

To predict the functions of IncRNAs, we constructed IncRNA-mRNA co-expression network(Fig.3).

\section{Construction of a ceRNA network.}

IncRNAs could function as ceRNA to competing the binding between miRNA and mRNA. Analysis of ceRNA network helped to understand the characterization of IncRNAs. As shown in Fig.4, 71 differentially expressed IncRNAs and 
123 expressed mRNAs were selected, which predicted 11 miRNAs sharing binding sites with the differentially expressed IncRNAs and mRNAs.

\section{Validation of differentially IncRNAs}

Nice differentially expressed IncRNAs from ceRNA network prediction were selected to confirm RNA sequence results by qRT-PCR(Table 1). Ten pairs of mice spleen which contain both T-ALL leukemia mice or C57BL/ 6 mice were selected. 10 downregulated IncRNAs $(\mathrm{p}<0.05 ; \mathrm{FC}>5$-fold $)$ were selected. All these InRNAs expression were downregulated and consistent with RNA sequence results $(P<0.05)$. Moreover, NONMMUT026003.2 was maximal changed IncRNAs in these IncRNAs.

\section{Discussion}

Notch1 is one of the major driving oncogene in T-ALL. About $55 \%$ of T-ALL patients occurred Notch1 mutation in the trans-membrane region and the intracellular PEST domain, which resulted abnormal activation of the Notch signaling pathway. Overexpression of ICN1 by retroviral infection in hematopoietic progenitor cells or thymocytes promote T ALL tumorigenesis, which was made to set up mouse T-ALL model. Over the past decades, the molecular mechanism of Notch1-correlated T-ALL has been extensively investigated. However, the precise pathogenesis of Notch1-correlated T-ALL is still unknown. Recent years, ncRNAs, including IncRNAs, have been found to be related with humorous number of biological regulatory functions. IncRNA NALT activating Notch signaling pathway promoted cell proliferation in T-ALL[13]. IncRNA-IUR acted as a tumor suppressors by suppressing the STAT5-CD71 pathway in Bcr-Abl-mediated tumorigenesis of T-ALL[14]. To further confirm the significant differences in the expression of IncRNAs and mRNAs, we removed the spleen from T-ALL leukemia mice and C57BL/ 6 mice and performed deep RNA sequence to study the different expression of IncRNAs and mRNAs. We found that IncRNAs' expression altered significantly in the spleen of Notch1-correlated T-ALL leukemia mice compared with that of C57BL/ 6 mice which was the first time to report the altered expression of IncRNAs. 1873 IncRNAs and 5626 mRNAs were differentially expressed in the spleen from T-ALL leukemia mice compared with that of C57BL/6 mice.

we performed GO terms enrichment to study the biological functions of differently expressed mRNAs. The most enriched GO terms for differentially expressed mRNAs were related to melanocyte differentiation, myosin complex and translation repressor activity in biological processes, cellular component or molecular function. To understand the function of differentially expressed mRNAs further, we performed KEGG pathway analysis and found that 303 pathways were significantly enriched among the altered transcripts. Acute myeloid leukemia, Protein export ad Glycosaminoglycan biosynthesis-keratan sulfate were the 3 significantly enriched pathways. Apoptosis, Notch signaling pathway and PI3K-Akt signaling pathway which was closely related with the pathology process of T-ALL was involved in the enriched pathways[15]. Furthermore, we constructed co-expression network to investigate the relation between IncRNAs and the coding genes.

Competing endogenous RNAs (ceRNAs) was raised that ceRNA molecules could sponge miRNA through miRNA response elements (MREs) and regulate gene expression. Numbers of molecules could act as ceRNAs, including IncRNAs, circRNAs or pseudogene. Wang et al found that IncRNA CHRF regulates cardiac hypertrophy by targeting miR-489[16]. circRNA MT01 sponges miR-9 to suppress hepatocellular carcinoma progression[17]. Chan et al found that A FTH1 gene:pseudogene, can sponge miRNAs to regulates tumorigenesis in prostate cancer[18]. In this study, a IncRNA-associated ceRNA network analysis was perform and showed that 71 differentially expressed IncRNAs and 123 expressed mRNAs were selected, which predicted 11 miRNAs sharing binding sites with the differentially expressed IncRNAs and mRNAs. 
Nice differentially expressed IncRNAs from ceRNA network prediction were selected to confirm deep RNA sequence results by qRT-PCR. We selected ten pairs of mice spleen which contain both T-ALL leukemia mice or C57BL/ 6 mice to further validation. All of the nice downregulated IncRNAs expression were consistent with RNA sequence results. NONMMUT117521.1 was located in intron of ECE-1 which has endopeptidase activity and membrane-bound metalloprotease. Bao et al found that ECE-1 promoted Ischemia/Reperfusion-Induced Injury[19]. ENSMUST00000195494 was located in Pfkfb3 which participated in the glucose metabolism and promoted cell proliferation, apoptosis and autophage in many types of cancer[20]. NONMMUT008951.2 was located in Bcl11a which inhibited proliferation and promoted apoptosis in B lymphoma cell lines[21]. NONMMUT026003.2, in the ceRNA network of differentially expressed IncRNAs, was located Bcl6 which participated humorous of processes, including inflammatory response, growth and differentiation[22].

This study provides the first Notch1-correlated ceRNA network prediction of IncRNAs and mRNAs which needed some studies to explore the roles of these differentially expressed IncRNAs. Because of the limitation of mice model, patients' samples addition is valuable.

\section{Abbreviations}

T-ALL Acute T-lymphocytic leukemia

ceRNA competing endogenous RNA

ICN Notch intracellular domain

GO Gene Ontology

KEGG Kyoto Encyclopedia of Genes and Genomes

MiRNA microRNA

qRT-PCR Quantitative real time polymerase chain reaction

NALT NOTCH1 associated IncRNA in T cell acute lymphoblastic leukemia 1

IUR Imatinib-upregulated IncRNA

STAT5 Signal transducer and activator of transcription 5

CD71 Cluster of Differentiation 71

PI3K Phosphatidylinositol 3 kinase

MREs miRNA response elements

MT01 mitochondrial tRNA translation optimization 1

CHRF Cardiac hypertrophy related factor

FTH1 Ferritin heavy chain 1

ECE-1 Endothelin converting enzyme 1 
Pfkfb3 6-phosphofructo-2-kinase/fructose-2,6-biphosphatase 3

\section{Declarations}

\section{Acknowledgements}

This study was supported by Department of Physiology, Hebei Medical University.

\section{Funding}

Supported by grants from Prevention and Treatment of Geriatric Diseases Funding of Finance of Hebei Province (No.[2018]772).

\section{Availability of data and materials}

RNA-seq data are included in additional files.

\section{Authors' contributions}

M.S prepared Figures.

S.W wrote manuscript text.

X.L prepared the tissues of animals.

Y.L. designed, directed the experiment and analyzed data and wrote the manuscript text.

All authors reviewed the manuscript.

\section{Ethics approval}

All animals were housed and cared for according to the guidelines and regulations of the Institutional Animal Care and Use Committee of the Second Hospital of Hebei Medical University.

\section{Consent for publication}

This work described was original research that has not been published previously, and not under consideration for publication elsewhere, in whole or in part.

\section{Competing interests}

The authors have declared that no competing interest exists.

\section{References}

1. Belver L, Ferrando A. The genetics and mechanisms of T cell acute lymphoblastic leukaemia. Nat Rev Cancer. 2016;16:494-507.

2. Hunger SP, Mullighan CG. Acute Lymphoblastic Leukemia in Children. N Engl J Med. 2015;373:1541-52.

3. Litzow MR, Ferrando AA. How I treat T-cell acute lymphoblastic leukemia in adults. Blood. 2015;126:833-841.

4. Pui CH, Robison LL, Look AT. Acute lymphoblastic leukaemia. Lancet. 2008;371:1030-43. 
5. Wang H, Zou J, Zhao B, Johannsen E, Ashworth T, Wong H, Pear WS, Schug J, Blacklow SC, Arnett KL, Bernstein BE, Kieff E, Aster JC. Genome-wide analysis reveals conserved and divergent features of Notch1/RBPJ binding in human and murine T-lymphoblastic leukemia cells. Proc Natl Acad Sci U S A. 2011;108:14908-13.

6. Jenkinson S, Koo K, Mansour MR, Goulden N, Vora A, Mitchell C, Wade R, Richards S, Hancock J, Moorman AV, Linch DC, Gale RE. Impact of NOTCH1/FBXW7 mutations on outcome in pediatric T-cell acute lymphoblastic leukemia patients treated on the MRC UKALL 2003 trial. Leukemia. 2013;27:41-7.

7. Sanchez-Martin M, Ferrando A. The NOTCH1-MYC highway toward T-cell acute lymphoblastic leukemia. Blood. 2017;129:1124-1133.

8. Brandimarte L, La Starza R, Gianfelici V, Barba G, Pierini V, Di Giacomo D, Cools J, Elia L, Vitale A, Luciano L, Bardi A, Chiaretti S, Matteucci C, Specchia G, Mecucci C. Mecucci.DDX3X-MLLT10 fusion in adults with NOTCH1 positive T-cell acute lymphoblastic leukemia. Haematologica. 2014;99:64-6.

9. Robles-Valero J, Lorenzo-Martín LF, Menacho-Márquez M, Fernández-Pisonero I, Abad A, Camós M, Toribio ML, Espinosa L, Bigas A, Bustelo XR. A Paradoxical Tumor-Suppressor Role for the Rac1 Exchange Factor Vav1 in T Cell Acute Lymphoblastic Leukemia. Cancer cell. 2017;32:608-623.

10. Misawa A, Takayama K, Urano T, Inoue S. Androgen-induced Long Noncoding RNA (IncRNA) SOCS2-AS1 Promotes Cell Growth and Inhibits Apoptosis in Prostate Cancer Cells. J Biol Chem. 2016;291:17861-80.

11. Kopp F, Mendell JT. Functional Classification and Experimental Dissection of Long Noncoding RNAs. Cell. 2018;172:393-407.

12. Xu J, Li Y, Lu J, Pan T, Ding N, Wang Z, Shao T, Zhang J, Wang L, Li X. The mRNA related ceRNA-ceRNA landscape and significance across 20 major cancer types. Nucleic Acids Res. 2015;43:8169-82.

13. Wang Y, Wu P, Lin R, Rong L, Xue Y, Fang Y. LncRNA NALT interaction with NOTCH1 promoted cell proliferation in pediatric T cell acute lymphoblastic leukemia. Scientific reports, 2015,5:13749.

14. Wang X, Yang J, Guo G, Feng R, Chen K, Liao Y, Zhang L, Sun L, Huang S, Chen JL. Novel IncRNA-IUR suppresses Bcr-Abl-induced tumorigenesis through regulation of STAT5-CD71 pathway. Mol Cancer. 2019;18:84.

15. Eric C, Hales,Jeffrey W, Taub,Larry H, Matherly. (2014). New insights into Notch1 regulation of the PI3K-AKTmTOR1 signaling axis: targeted therapy of $y$-secretase inhibitor resistant T-cell acute lymphoblastic leukemia. Cellular signaling 26:149-61.

16. Wang K, Liu F, Zhou LY, Long B, Yuan SM, Wang Y, Liu CY, Sun T, Zhang XJ, Li PF. The long noncoding RNA CHRF regulates cardiac hypertrophy by targeting miR-489. Circ Res. 2014;114:1377-88.

17. Han D, Li J, Wang H, Su X, Hou J, Gu Y, Qian C, Lin Y, Liu X, Huang M, Li N, Zhou W, Yu Y, Cao X. Circular RNA circMT01 acts as the sponge of microRNA-9 to suppress hepatocellular carcinoma progression. Hepatology. 2017;66:1151-1164.

18. Chan JJ, Kwok ZH, Chew XH, Zhang B, Liu C, Soong TW, Yang H, Tay Y. A FTH1 gene:pseudogene:microRNA network regulates tumorigenesis in prostate cancer. Nucleic Acids Res. 2018;46:1998-2011.

19. Bao N, Fang B, Lv H, Jiang Y, Chen F, Wang Z, Ma H. Upregulation of miR-199a-5p Protects Spinal Cord Against Ischemia/Reperfusion-Induced Injury via Downregulation of ECE1 in Rat. Cell Mol Neurobiol. 2018;38:12931303.

20. O'Neal J, Clem A, Reynolds L, Dougherty S, Imbert-Fernandez Y, Telang S, Chesney J, Clem BF. Inhibition of 6phosphofructo-2-kinase (PFKFB3) suppresses glucose metabolism and the growth of HER2+ breast cancer. Breast Cancer Res Treat. 2016;160:29-40. 
21. Gao Y, Wu H, He D, Hu X, Li Y. Downregulation of BCL11A by siRNA induces apoptosis in B lymphoma cell lines. Biomed Rep. 2013;1:47-52.

22. Leeman-Neill RJ, Bhagat G. BCL6 as a therapeutic target for lymphoma. Expert Opin Ther Targets. 2018;22:143152.

\section{Tables}

Table 1 Basic information of selected nice IncRNAs for qRT-PCR confirmation.

\begin{tabular}{|c|c|c|c|c|c|c|}
\hline Seqname & Source & $\begin{array}{l}\text { RNA } \\
\text { length }\end{array}$ & Locus & P-value & $\begin{array}{l}\text { Fold } \\
\text { change }\end{array}$ & Regulation \\
\hline NONMMUT117521.1 & PMID24463510 & 863 & $\begin{array}{l}4: 137882371- \\
137883938\end{array}$ & 0.007679055 & 7.33 & DOWN \\
\hline NONMMUT021309.2 & PMID23951020 & 2508 & $\begin{array}{l}14: 65114738- \\
65117245\end{array}$ & 0.005231906 & 5.65 & DOWN \\
\hline ENSMUST00000195494 & noncodeV3 & 2099 & $\begin{array}{l}2: 11532596- \\
11534694\end{array}$ & 0.000178499 & 10.60 & DOWN \\
\hline NONMMUT008951.2 & noncodeV3 & 3828 & $\begin{array}{l}11: 24103379- \\
24107440\end{array}$ & 4.34E-05 & 12.53 & DOWN \\
\hline NONMMUT008779.2 & noncodeV3 & 3379 & $\begin{array}{l}11: 20461259- \\
20464664\end{array}$ & 0.00264931 & 9.67 & DOWN \\
\hline NONMMUT026003.2 & noncodeV3 & 2078 & $\begin{array}{l}16: 23981313- \\
23983390\end{array}$ & 0.004331438 & 5.59 & DOWN \\
\hline NONMMUT041921.2 & NONCODEv4 & 3925 & $\begin{array}{l}2: 181405971- \\
181409895\end{array}$ & 0.000122807 & 7.66 & DOWN \\
\hline NONMMUT147972.1 & - & 1666 & $\begin{array}{l}\text { 4:32239436- } \\
32241101\end{array}$ & 0.001742802 & 8.78 & DOWN \\
\hline NONMMUT029828.2 & NONCODEv3 & 2875 & $\begin{array}{l}17: 46424215- \\
46427089\end{array}$ & 3.45E-05 & 32.22 & DOWN \\
\hline
\end{tabular}

Table 2 Specific primers of each IncRNA. 


\begin{tabular}{|lll|}
\hline Seqname & Forward & Reverse \\
\hline NONMMUT117521.1 & GATGCTGAGCAAGTGGGTGA & TGGATAGGTGGATGTGAGCG \\
\hline NONMMUT021309.2 & TCCAACATTGCTCACCCGAG & AGCATGGCGCTGTCAATGTA \\
\hline ENSMUST00000195494 & GGATCTGCAGAGGGGCTTAC & GCACCAGGGAACCCATTGTA \\
\hline NONMMUT008951.2 & CAGGACGAGTCAGGCACATT & CAAGCCTTCGGTCTGTGTCT \\
\hline NONMMUT008779.2 & AGTCCTTGTGTGAGCATCCG & CCCATGTGTTCCGTGGAGAA \\
\hline NONMMUT026003.2 & AAGAGGCTGGCTAGACCTGA & AAGGAGTTTGAAGGCGGGAG \\
\hline NONMMUT041921.2 & AAGATGTAAGGGCCACCAGC & TGTGAGGCTGTTCCAAGTCC \\
\hline NONMMUT147972.1 & ACCCAACAAGATGCTCCTCG & AAGTCGCAGTTGGGTCTCTG \\
\hline NONMMUT029828.2 & TTCAGGTTCAGCTGTTCGGG & GAGTGGGGAGCCGTAAGAAC \\
\hline
\end{tabular}

\section{Figures}


A

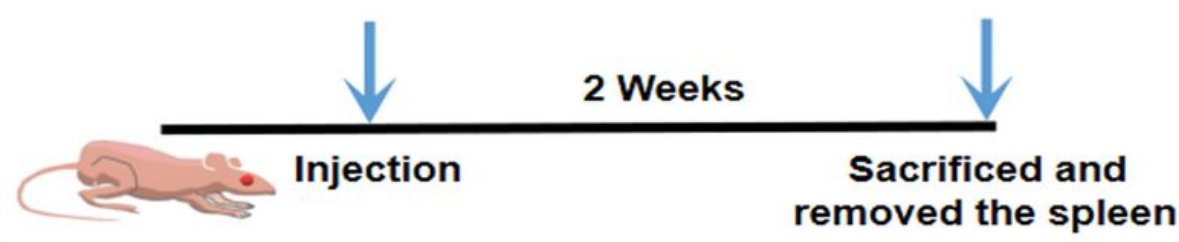

B

D
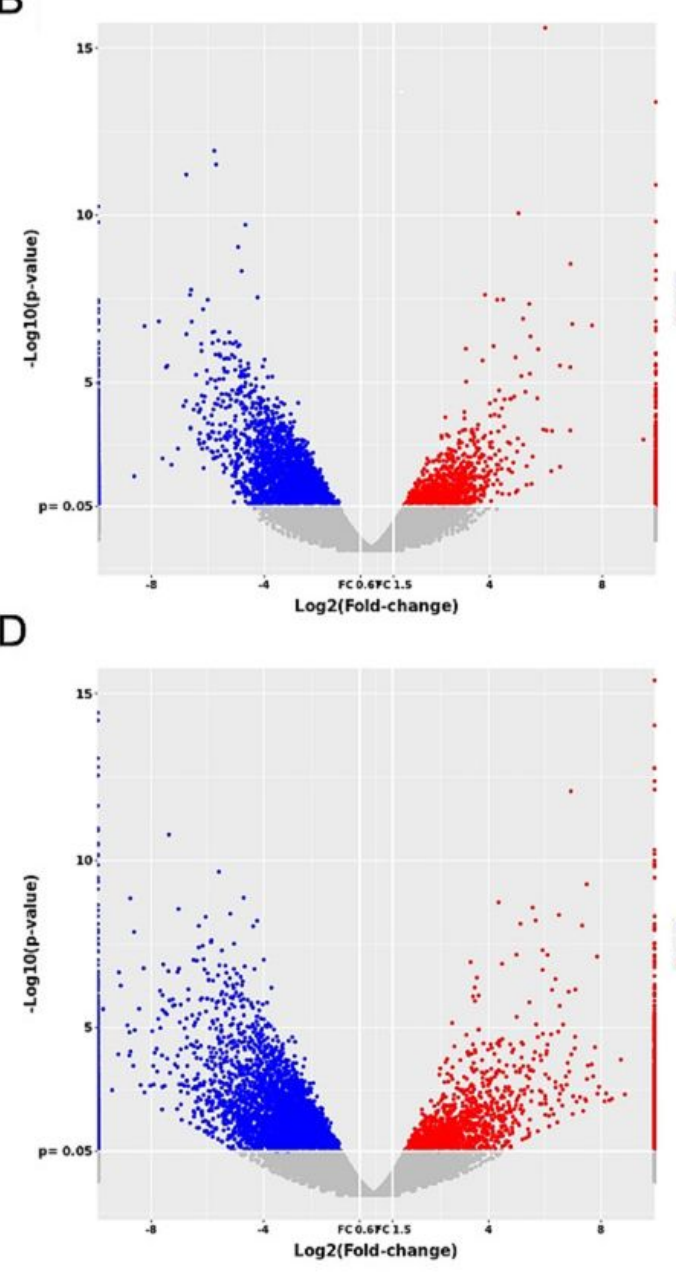

C

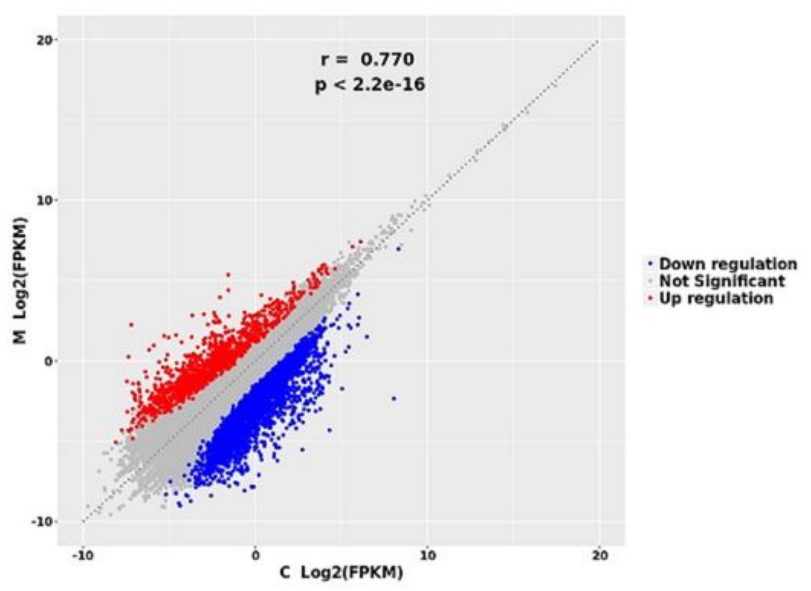

$\mathrm{E}$

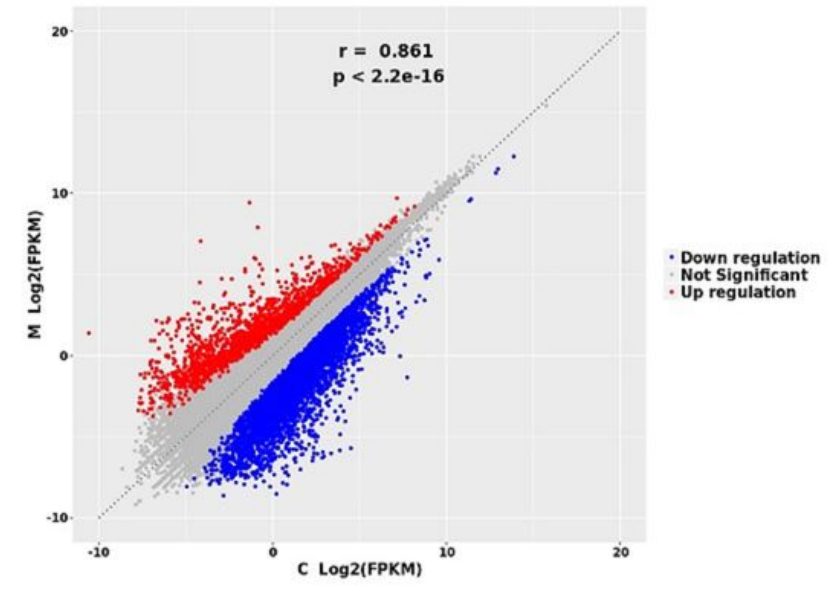

Figure 1

Differentially expressed IncRNAs and mRNAs in spleen of mice. The time-line of study design (A). Differentially expressed IncRNAs are shown in volcano Plot(B) 16 and scatter-Plot(C). Differentially expressed mRNAs are shown in volcano plot(D) and scatter plot(E). For volcano plot, the abscissa is fold change(fold change $\geq 2$ ) and the ordinate is $p$ value ( $p$-value $\leq 0.05$ ). The red color is the up-regulated genes and the blue is the down-regulated genes. For scatter plot, The abscissa and ordinate represent two sets of samples. The red color is the up-regulated genes and the blue is the down-regulated genes. 
A

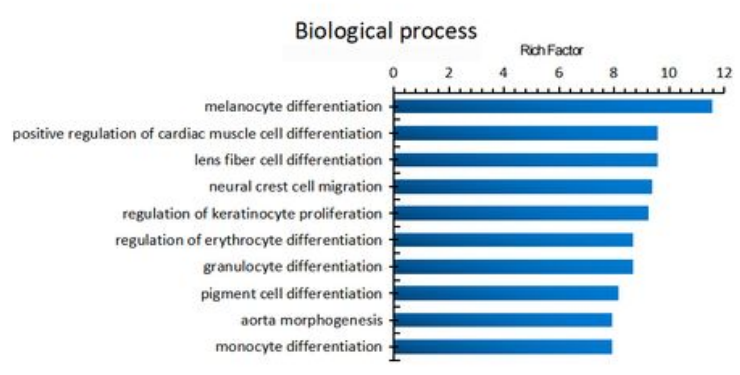

C

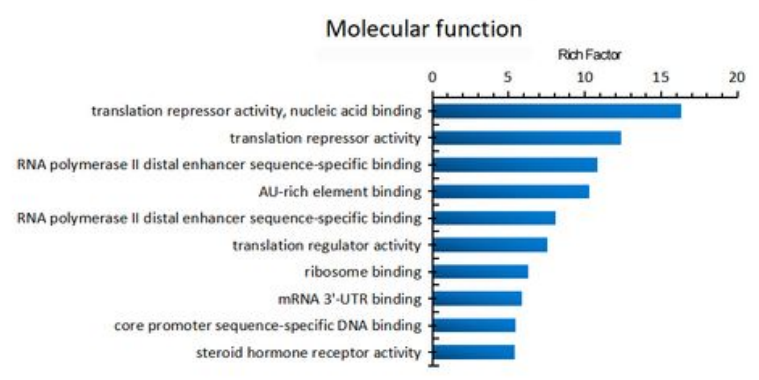

B
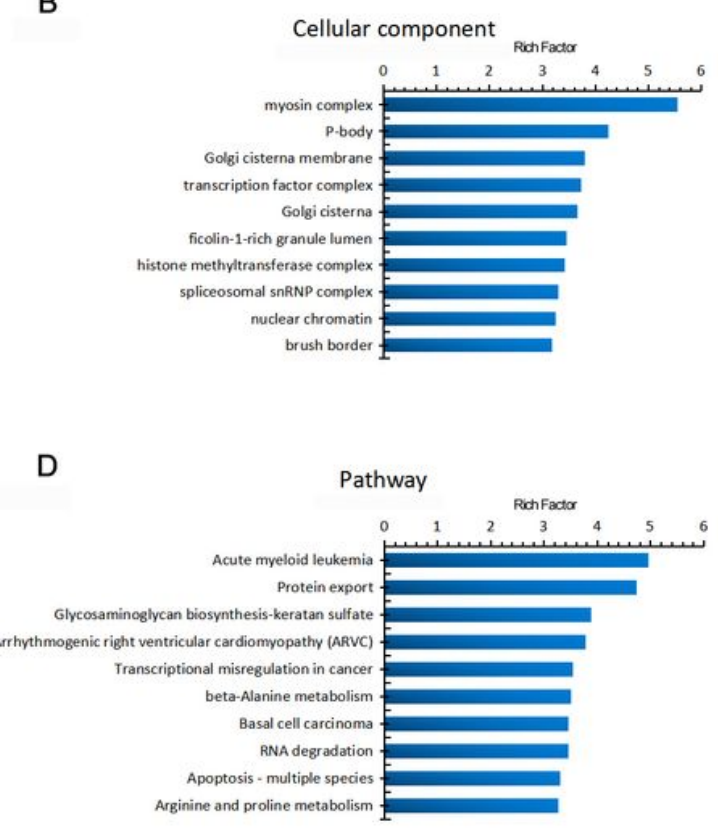

\section{Figure 2}

mRNA GO and KEGG pathway analysis in spleen of mice. TOP10 enriched GO terms for Biological process(A), cellular component(B) and molecular function(C). TOP10 enriched KEGG pathway was shown in corresponded to differentially expressed transcripts(D). 


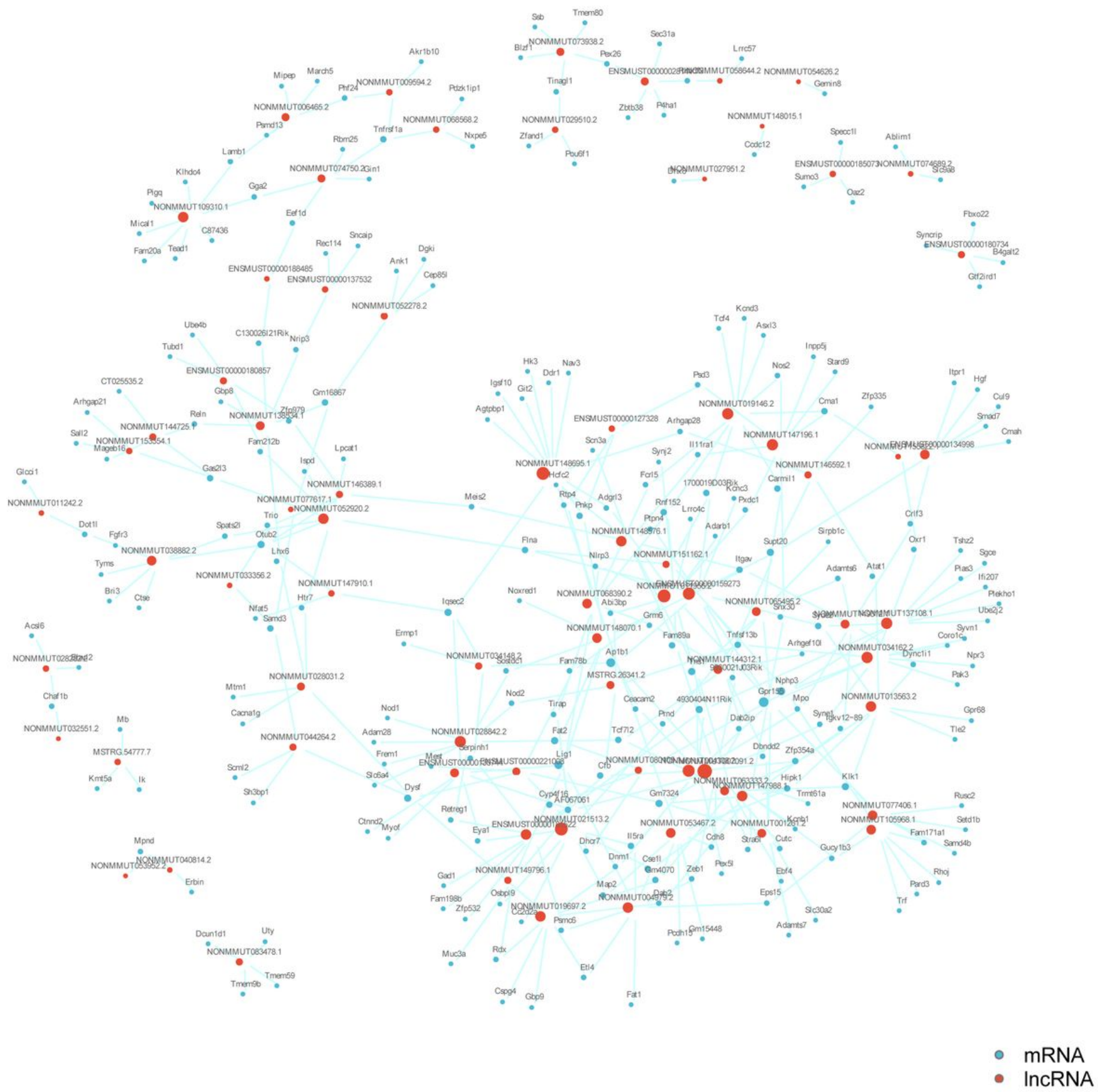

Figure 3

IncRNAs and mRNAs co-expression analysis. This network shown the co-expression correlations between IncRNAs and mRNAs. 

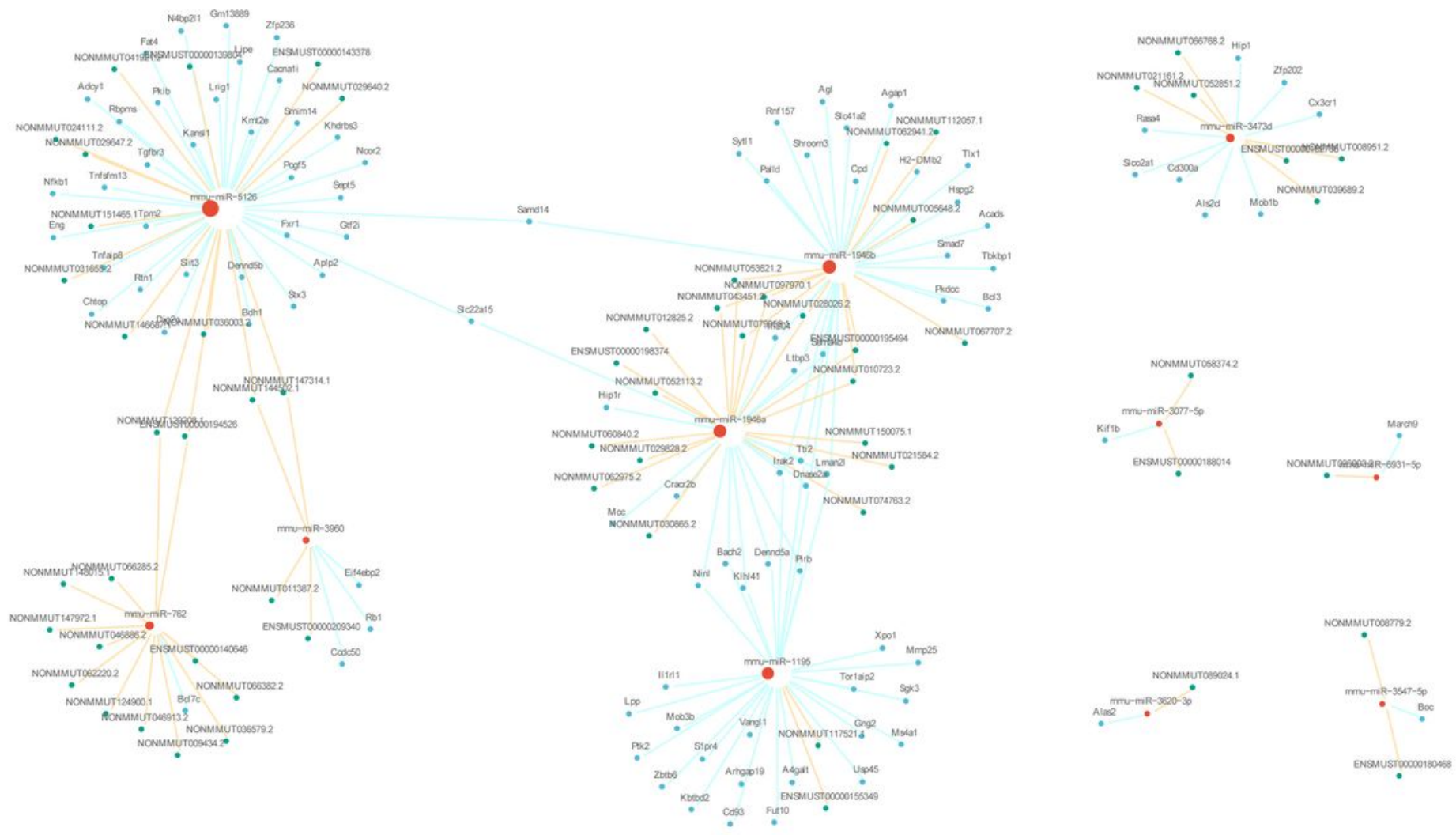

Figure 4

Construction of a ceRNA network. Global view of the ceRNA network. Six networks were presented. 

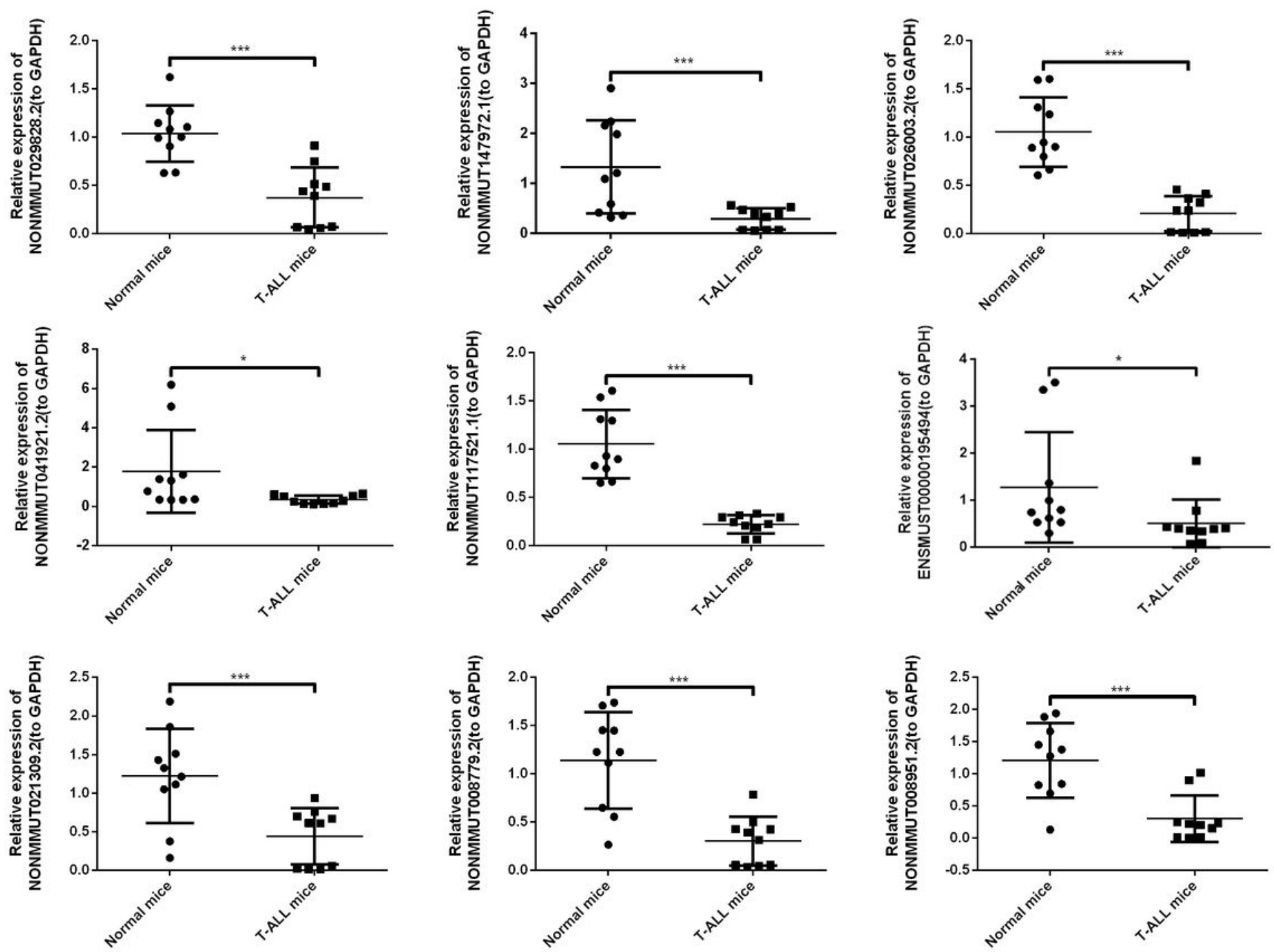

Figure 5

Validation of deep RNA sequence results by qRT-PCR from spleen of T-ALL mice or C57BL/6 mice.

\section{Supplementary Files}

This is a list of supplementary files associated with this preprint. Click to download.

- additionaldata.xls

- ARRIVEGuidelinesChecklist.pdf 